\title{
BRAF activates and physically interacts with PAK to regulate cell motility
}

\author{
Samantha K McCarty ${ }^{1}$, Motoyasu Saji ${ }^{1}$, Xiaoli Zhang ${ }^{3}$, Christina M Knippler ${ }^{1}$, \\ Lawrence S Kirschner ${ }^{1,2}$, Soledad Fernandez ${ }^{3}$ and Matthew D Ringel ${ }^{1,2}$ \\ ${ }^{1}$ Division of Endocrinology, Diabetes and Metabolism, Department of Internal Medicine, ${ }^{2}$ Department of \\ Molecular Virology, Immunology, and Medical Genetics, ${ }^{3}$ Center for Biostatistics, The Ohio State University and \\ Arthur G. James Comprehensive Cancer Center, 565 McCampbell Hall, 1581 Dodd Dr., Columbus, Ohio 43210, USA
}

Correspondence should be addressed to M D Ringel

Email matthew.ringel@osumc.edu

\begin{abstract}
Increased p21-activated kinase (PAK) signaling and expression have been identified in the invasive fronts of aggressive papillary thyroid cancers (PTCS), including those with RETIPTC, BRAFV600E, and mutant RAS expression. Functionally, thyroid cancer cell motility in vitro is dependent on group 1 PAKs, particularly PAK1. In this study, we hypothesize that BRAF, a central kinase in PTC tumorigenesis and invasion, regulates thyroid cancer cell motility in part through PAK activation. Using three well-characterized human thyroid cancer cell lines, we demonstrated in all cell lines that BRAF knockdown reduced PAK phosphorylation of direct downstream targets. In contrast, inhibition of MEK activity either pharmacologically or with siRNA did not reduce PAK activity, indicating MEK is dispensable for PAK activity. Inhibition of cell migration through BRAF loss is rescued by overexpression of either constitutive active MEK1 or PAK1, demonstrating that both signaling pathways are involved in BRAF-regulated cell motility. To further characterize BRAF-PAK signaling, immunofluorescence and immunoprecipitation demonstrated that both exogenously overexpressed and endogenous PAK 1 and BRAF co-localize and physically interact, and that this interaction was enhanced in mitosis. Finally, we demonstrated that acute induction of BRAFV600E expression in vivo in murine thyroid glands results in increased PAK expression and activity confirming a positive signaling relationship in vivo. In conclusion, we have identified a signaling pathway in thyroid cancer cells which BRAF activates and physically interacts with PAK and regulates cell motility.
\end{abstract}

\section{Key Words}

- thyroid cancer

- cell motility

- PAK1

- BRAF

- cancer biology

\section{Introduction}

Over the past three decades, the incidence of thyroid cancer, particularly papillary thyroid cancer (PTC) has been increasing. While this rise is in large part due to improved diagnostics and early detection, the occurrence of larger tumors and the annual number of individuals who die from thyroid cancer are rising (Pellegriti et al. 2013). Most thyroid cancers are curable if detected at an early stage; however, for patients with aggressive forms of thyroid cancer treatment options are limited and prognosis is poor (Ringel 2009). Predictors of death include the presence of gross local invasion and the presence of larger distant metastases (Wong et al. 2013). Defining the mechanisms underlying thyroid cancer development, invasion, and metastases is important in order to develop effective targeted therapies.

Published by Bioscientifica Ltd 
PTC tumorigenesis is driven largely by activation of the MAP kinase pathway through several genetic events, the most common of which are activating mutations in $B R A F$ (most frequently BRAFV600E), RAS isoforms, or gene rearrangements involving the RET tyrosine kinase (Fagin \& Mitsiades 2008, Nucera et al. 2011). Whether activated by WT BRAF signaling through RAF homodimers or heterodimers, or by monomeric signaling from constitutively activated (CA) BRAFV600E, activated MEK leads to a downstream cascade of signaling events resulting in increased proliferation, invasiveness, reduced apoptosis, and reduced tissue-specific function (Knauf et al. 2005, Melillo et al. 2005, Riesco-Eizaguirre et al. 2009). BRAFV600E has been particularly well studied in PTC. Expression of BRAFV600E in the mouse causes thyroid cancer and thyroid cell dedifferentiation in vivo (Knauf et al. 2005, 2011) and occurs in $\sim 40 \%$ of all human PTC samples, although this prevalence varies depending on geographic location and ethnicity. BRAFV600E in PTC is associated with more aggressive clinical behavior (Xing 2007, Xing et al. 2013), and recent data suggests that other genetic abnormalities, such as mutations in the hTERT promoter, may cooperate with BRAFV600E resulting in more aggressive tumor behavior (Landa et al. 2013, Liu et al. 2014). The frequency of BRAFV600E is also reported to be high in tumors from patients with progressive PTC enrolled in clinical trials (Kloos et al. 2009). For these reasons, there have been major efforts to evaluate the efficacy of inhibiting BRAF and/or MEK in thyroid and other BRAF-mediated cancers. This has resulted in FDAapproval of compounds targeting either all RAF isoforms or BRAFV600E specifically. However, these treatments are not curative and acquired resistance is nearly universal. Strategies to increase the effectiveness of BRAF-targeted compounds and to alleviate mechanisms of acquired resistance are being studied.

As gross tumor invasion predicts poor prognosis in thyroid cancer, we evaluated expression profiles of the invasive fronts of large invasive PTCs to identify potential therapeutic targets. This work demonstrated that PTC invasion was associated with signaling leading to epithelial-to-mesenchymal transition (Vasko et al. 2007). The studies implicated known thyroid cancer pathways such as PI3K and transforming growth factor beta (TGF $\beta$ ) signaling cascades in the invasive fronts, but also suggested a previously undefined role for p21-activated kinases (PAKs). Subsequently, we confirmed that PAK expression and phosphorylation were increased in the invasive fronts of aggressive PTCs and occurred in tumors with MAPKactivating genetic alterations. We further demonstrated that inhibition of group 1 PAKs (PAK1 in particular) reduced motility in six different human thyroid cancer cell lines (McCarty et al. 2010).

PAKs are a family of serine/threonine kinases that phosphorylate downstream targets that alter cell motility by regulating cytoskeletal proteins involved in promoting lamellopod extension, enhancing proliferation, and inhibiting apoptosis (Radu et al. 2014). PAKs play important roles in breast cancer development and progression (Shrestha et al. 2012, Rider et al. 2013), in schwannoma development as effectors of NF2 (Flaiz et al. 2009) and in neurological syndromes (Ma et al. 2012). The six isoforms of PAK are divided into group 1 (PAKs 1-3) and group 2 (PAKs 4-6) based on structural and functional similarities (Radu et al. 2014). RAC1 and CDC42 are the primary activators of group 1 PAKs (Radu et al. 2014) that normally exist as inactive homodimers through the binding of the auto inhibitory domain of one kinase to the kinase domain of another (Whale et al. 2011). When RAC1 and CDC42 bind to PAK, the homodimer relaxes allowing for activation (Lei et al. 2005). Once activated, PAKs phosphorylate downstream effectors including vimentin, cRAF, ROCK, and many others (Radu et al. 2014).

The relationship between PAK and RAF/MEK signaling is complex. PAK is known to phosphorylate CRAF and MEK-enhancing activation, suggesting a potentiating role for PAK in RAF and MEK signaling (Slack-Davis et al. 2003, Wang et al. 2013, Radu et al. 2014). In addition to their kinase activity, group 1 PAKs have a kinase-independent scaffold function that sequesters CRAF and MEK1 at the plasma membrane enhancing signaling (Wang et al. 2013). Similar scaffolding functions allow PAK1 to promote AKT signaling (Higuchi et al. 2008). PAK1 coordinately activates MET and MAPK signaling in breast cancer cells and PAK1 amplification has been proposed to be an alternative pathway for MAPK activation in this tissue type (Shrestha et al. 2012). It is of interest that in melanoma tissue microarrays (presumably from central tumor cores), PAK1 expression levels are lower in BRAFV600E-positive tumors vs WT tumors (Ong et al. 2011). Concordantly, PAK1 mediates MAPK activation in melanoma cells with WT BRAF more so than in those homozygous for BRAFV600E through phosphorylation of CRAF and MEK (Ong et al. 2011). However, a role for BRAF beyond kinase inhibition (i.e. through scaffolding or other mechanisms) or the mechanism for its regulation of PAK function has not been reported. In addition, the regulation of PAK by MAPK signaling proteins has not yet been reported in thyroid cancer cells that have unique resistance mechanisms to BRAF inhibitors (Montero-Conde et al. 2013).

Published by Bioscientifica Ltd 
In this study, we demonstrate in thyroid cancer cells that PAK activity is dependent on BRAF expression. We also demonstrate that MEK does not regulate PAK signaling and report the new observation that BRAF and PAK physically interact. Interestingly, the endogenous BRAF-PAK1 interaction appears to be enhanced in mitosis. Finally, in vivo acute overexpression of BRAFV600E results in increased PAK expression and activity in addition to ERK phosphorylation. These data together are consistent with the conclusion that PAK is a MEK-independent functional downstream effector of BRAF that may play a role in thyroid tumorigenesis and progression.

\section{Materials and methods}

\section{Cell culture}

Human thyroid carcinoma BCPAP, TPC1, and FTC133 cell lines (heterozygous for BRAFV600E; express RET/PTC1, or are BRAF WT respectively) were the generous gifts of Drs R Schweppe (University of Colorado Denver) (Schweppe et al. 2008) with permission from the researchers who originally established cell lines: FTC133 - P Goretzki, University of Leipzig, Germany (Goretzki et al. 1990); BCPAP - D N Fabien, Centre Hospitalier Lyon-Sud, France (Fabien et al. 1994); and TPC1 - H Sato, Kanazawa University, Japan (Kurebayashi et al. 2000). The obtained cell lines were independently confirmed to be of thyroid origin by DNA fingerprinting using methods as previously described (Schweppe et al. 2008). The cells were cultured as described (McCarty et al. 2010). Human embryonic kidney (HEK) 293 cells were purchased from the American Type Culture Collection (ATCC, Manassas, VA, USA) and grown in conditions described (Ding et al. 2013).

\section{Cell synchronization}

TPC1 cells maintained at low confluency were treated with $2 \mathrm{mM}$ of thymidine in DMEM with $10 \%$ FBS for $24 \mathrm{~h}$, released for $2 \mathrm{~h}$, and treated with $100 \mathrm{ng} / \mathrm{ml}$ of nocodazole in DMEM with $10 \%$ FBS for $24 \mathrm{~h}$. TPC1 cells were released for $1 \mathrm{~h}$ and protein was isolated using the method described below.

\section{cDNA constructs}

Vectors containing the cDNAs encoding CA PAK1 and GFP-tagged PAK autoinhibitory domain (PID) were the generous gifts of J Chernoff (Fox Chase Cancer Center) (Beeser \& Chernoff 2005). The vector containing the
cDNA for murine CA MEK1 was a gift from M Ostrowski (The Ohio State University, Columbus OH, USA; Huang $\&$ Erikson 1994). The vector containing the cDNA for MYC-tagged BRAF was a gift from Dr James Fagin (Memorial Sloan-Kettering Cancer Center, New York, NY, USA). The FLAG-PAK1 vector was constructed by excising cDNA from a WT PAK1 vector using BamHI and HindIII. The fragment underwent PCR to add a FLAG-tag and was then inserted into the $\mathrm{pCMV}-\mathrm{Tag} 2 \mathrm{~B}$ vector.

\section{Transient transfections}

CA PAK1 cDNA, CA MEK1 cDNA, PID cDNA, and BRAF siRNA combinations were transfected into BCPAP, TPC1, and FTC133 cells using Optifect Reagent (Life Technologies Co.; McCarty et al. 2010). HEK293 cells were transfected with MYC-tagged BRAF, FLAG-tagged PAK1, and HA control using Lipofectamine Plus (Life Technologies Co.) as previously described (Porchia et al. 2007).

\section{siRNA transfections}

BCPAP, TPC1, and FTC133 cells were grown to $30-40 \%$ confluence and transfected with scrambled siRNA control (cat no. sc-37007, Santa Cruz Biotechnology, Inc.), BRAF-specific siRNAs (cat no. sc-36368, Santa Cruz Biotechnology, Inc.), PAK1-specific siRNAs (cat no. sc-29700, Santa Cruz Biotechnology, Inc.), MEK1-specific siRNAs (cat no. sc-29396, Santa Cruz Biotechnology, Inc.), or MEK2 (cat no. sc-35905, Santa Cruz Biotechnology, Inc.) using Lipofectamine 2000 (Life Technologies Co.) as previously described (McCarty et al. 2010).

\section{U0126 treatment}

BCPAP, TPC1, and FTC133 cells were grown to 50\% confluence and treated with U0126 (Cell Signaling Technology, Danvers, MA, USA) at various concentrations for $24 \mathrm{~h}(0-10 \mu \mathrm{M})$. For migration experiments involving U0126, the cells were seeded, allowed to settle for $1 \mathrm{~h}$, and incubated with U0126 or DMSO $(2 \mu \mathrm{M})$ for $2 \mathrm{~h}$ before being exposed to a serum gradient.

\section{Protein isolation and western blotting}

Protein was isolated using either M-PER or lysis buffer and then Western blot was performed as previously described (Ringel et al. 2001, Vasko et al. 2007). Primary antibodies against PAK1, phospho-Thr423 PAK1/Thr402 PAK2, cRAF, phospho-Ser338 cRAF, MEK1/2, phospho-Ser217

Published by Bioscientifica Ltd. 
MEK1/Ser221MEK2, phospho-Ser298 MEK1, LIMK1, LIMK2, phospho-Thr508 LIMK1/Thr505LIMK2, ERK1/2, phospho-Thr202 ERK1/Thr204 ERK2, MYC-TAG, and GAPDH were from Cell Signaling Technology and antibodies against BRAF and GFP were from Santa Cruz Biotechnology, Inc. The antibody against vimentin and phospho-Ser55 vimentin were from Sigma-Aldrich, Inc. and MBL Co. (Nagoya, Japan) respectively.

\section{Migration assay}

Migration assays were performed on BCPAP, FTC133, and TPC1 cells as previously described (McCarty et al. 2010).

\section{Cell viability analysis}

The effect of transfection and U0126 treatment on cell viability was assessed using the 3-(4,5-dimethylthiazol-2-yl) -2,5-diphenyl-2H-tetrazolium bromide (MTT) assay as previously described (Porchia et al. 2007).

\section{Immunofluorescence and confocal microscopy}

HEK293 cells were grown on glass cover slips in six-well dishes, transfected, fixed with $4 \%$ paraformaldehyde (Affymetric, Cleveland, OH, USA), permeabilized with $0.1 \%$ Triton X-100, and incubated for 15 min in Vectastain (Vector Laboratories, Inc., Burlingame, CA, USA) blocking solution. Primary rabbit FLAG-Tag and mouse BRAF antibodies were applied overnight at $4{ }^{\circ} \mathrm{C}$. After washing twice with PBS, the cells were incubated with Alexa Fluor 488 and Alexa Fluor 594 secondary antibodies in PBS for $1 \mathrm{~h}$ in the dark. The cover slips were mounted onto the slides with Slow Fade Antifade reagent with DAPI (Life Technology Co.), dried in the dark for $2 \mathrm{~h}$, and stored in the dark at $4{ }^{\circ} \mathrm{C}$. The images were collected with a single-photon Olympus Flowview 1000 Laser Scanning Confocal microscope. TPC1 and FTC133 cells underwent the same process except they were not transfected and were seeded in a Labteck Chamber slide (Sigma). Primary antibodies against PAK1 and phospho-Thr423 PAK1/Thr402 PAK2 (\#2601) were used.

\section{Immunoprecipitation}

$200-400 \mu \mathrm{g}$ total protein weres incubated with preconjugated EZview Red anti-FLAG M2 affinity gel (Sigma), EZview Red anti-c-MYC affinity gel (Sigma), EZview Red anti-HA affinity gel (Sigma), non-conjugated anti-PAK1 antibody from Cell Signaling Technology, or non-conjugated anti-BRAF antibody from Santa Cruz
Biotechnology, Inc. overnight at $4{ }^{\circ} \mathrm{C}$. For the PAK1 and BRAF immunoprecipitation (IP), 1/10 volume of protein $\mathrm{G}$ agarose beads was added for $2 \mathrm{~h}$ to eliminate non-specific binding. The beads were centrifuged and the supernatant was collected for IP. The same amount of protein $G$ agarose beads was used as for the IP. Both mixtures were centrifuged and the pellets were washed with M-PER buffer three times. Loading buffer for Western blot (one volume of dye and three volumes of distilled water) was added and the mixtures were boiled to release the bound proteins and western blotting analysis was performed. For experiments using preconjugated beads, control experiments were included, in which preconjugated anti-hemaglutinin antibody beads was used for IP; for non-preconjugated bead experiments, IgG precipitation was used as the negative control.

\section{Immunohistochemical staining and western blotting of mouse thyroid tissue}

Paraffin blocks from the thyroid glands of mice with thyroid-specific, doxycycline-inducible BRAFV600E (iBRAFV600E) following 1 week of induction and WT control littermates were the generous gifts of J Fagin (Memorial Sloan Kettering Cancer Center, NY, USA; Chakravarty et al. 2011). The blocks were cut into sections, which were placed on slides and soaked in xylene followed by 100 and $95 \%$ ethanol. Endogenous peroxidase was quenched using 3\% peroxidase. The slides were prepared by heating in Antigen Unmasking Solution for 7 min (Vector Laboratories, Inc.). For blocking and primary antibody staining, immunostaining racks and cover plates (Thermo Scientific, Inc., Pittsburg, PA, USA) were used. The primary antibodies against Thr423 pPAK, PAK1, PAK2, and PAK3 were diluted, 1:100, in PBS and incubated on the slides overnight at $4{ }^{\circ} \mathrm{C}$. After the primary antibody, the slides were treated with a secondary antibody followed by streptavidin/peroxidase preformed complex solution and a DAB Substrate Mixture (Vector Laboratories, Inc.). They were then immersed in hematoxylin for $30 \mathrm{~s}$ and coverslips were mounted using $30 \%$ glycerol. Negative controls were slides that underwent the same process without the primary antibody. Immunohistochemistry (IHC) was scored based on the number of positive cells and the intensity of the immune activity independently by three investigators (S K McCarty, M Saji and V Vasko) on a 0-2 scale. Scoring was compared and averages were calculated.

For western blotting analysis, we also isolated mouse thyroid tissues from iBRAFV600E mice as well as age matched WT and rtTA-expressing control mice after

Published by Bioscientifica Ltd. 
1 week treatment with doxcycline-impregnated food pellets (2500 p.p.m.) as previously described (Chakravarty et al. 2011). The thyroid tissue was homogenized in M-PER buffer and protein was isolated from individually treated iBRAFV600E mice. For the treated WT and rTTA control mice, pooled thyroid gland tissue from each genotype group (due to the small thyroid size) was homogenized with M-PER buffer and protein was isolated. The proteins were concentrated using Amicon Ultra-0.5 Centrifugal Filter Devices (EMD Millipore Co., Billerica, MA, USA), concentrations were measured as above, and western blotting analyses were carried out. The mouse studies were approved by the Institutional Animal Care and Use Committee.

\section{Statistical analyses}

For cell migration studies, to investigate whether inhibition of BRAF and MEK1/2 (compared to control) would affect cell migration, linear mixed models were used to account for the correlation among observations from the same experiment. For the MTT assays, the data were first log-transformed to reduce variance. For the migration rescue experiments, linear mixed models with an interaction effect were used to analyze whether introducing either CA PAK1 or CA MEK1 into BRAF siRNA transfected cells rescued the effect of decreased migration caused by the BRAF siRNA. As a secondary analysis, an interaction between the interaction term and cell lines was used to test whether the rescue effect differed between cell lines. All analyses were performed using SAS/STAT Software version 9.2 (SAS Institute, Inc., Cary, NC, USA). To quantify the co-localization of BRAF with PAK1 and pPAK, the confocal images were analyzed using Pearson's coefficient of correlation $(r)$. A coefficient of 1 or -1 implies perfect positive or negative correlation, while 0 implies none. The Pearson's coefficient can then be converted into a percentage that represents the amount of overlap or co-localization. Complete details of the methods and mathematics are as reported previously (Zinchuk et al. 2007, French et al. 2008).

\section{Results}

\section{PAK function is regulated by BRAF}

To determine whether PAK function is dependent on BRAF expression, BRAF or control siRNA was transfected into the human thyroid cancer cell lines BCPAP, TPC1, and FTC133 that harbor different thyroid cancer-causing genetic mutations (see 'Materials and methods' section).
Western blotting analysis was carried out using specific antibodies for known PAK phosphorylation sites on vimentin (Ser56) and MEK (Ser298). In all cases, partial BRAF suppression was sufficient to reduce $P A K$-mediated phosphorylation of downstream targets as well as BRAFmediated MEK phosphorylation (Fig. 1A). In contrast, when group 1 PAK activity was specifically suppressed using exogenous PAK autoinhibitory domain (PID), no effect was observed on BRAF-mediated MEK phosphorylation despite reductions in $P A K$-mediated phosphorylation (Fig. 1B).

\section{BRAF-regulated thyroid cancer cell motility is dependent on MEK and PAK1}

Both BRAF and PAKs regulate cancer cell motility (McCarty et al. 2010, Arozarena et al. 2011, Makrodouli et al. 2011). BRAF siRNA blocked serum gradient-induced BCPAP, TPC1, and FTC133 thyroid cancer cell migration compared with the scrambled control in all three-cell lines (Fig. 2A). To ensure that BRAF suppression had a lesser effect on cell viability in the low confluency conditions

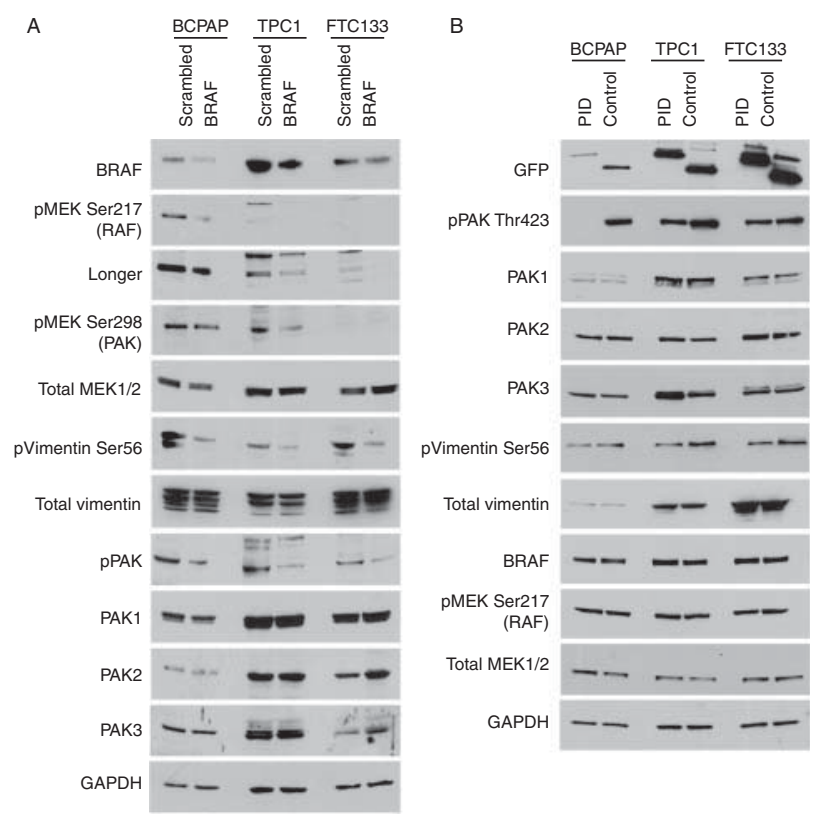

Figure 1

PAK function is BRAF dependent. (A) BRAF siRNA blocked PAK-mediated phosphorylation of vimentin and MEK in BCPAP, TPC1, and FTC133 cell lines vs scrambled sequence control. Transfection efficiencies were $\sim 40-60 \%$. 'Longer' refers to a longer exposure of the same blot for adequate visualization of the bands of all cell lines for the pMEK immunoblot (B) Expression of a molecular inhibitor of group 1 PAKs, GFP-tagged PID, did not reduce BRAF-mediated MEK1/2 phosphorylation in all three-thyroid cancer cell lines vs vector transfection control. Representative western blottings are shown for experiments repeated independently on at least two occasions.

Published by Bioscientifica Ltd. 

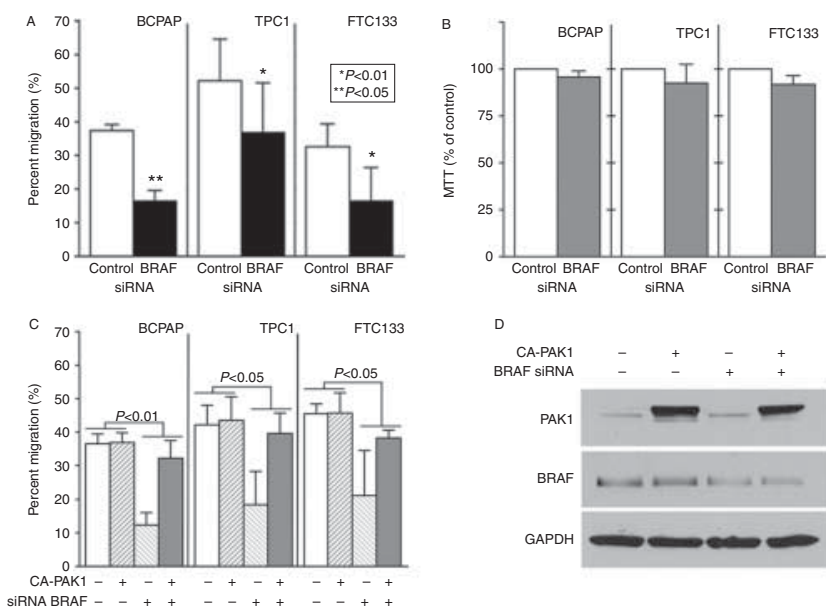

D
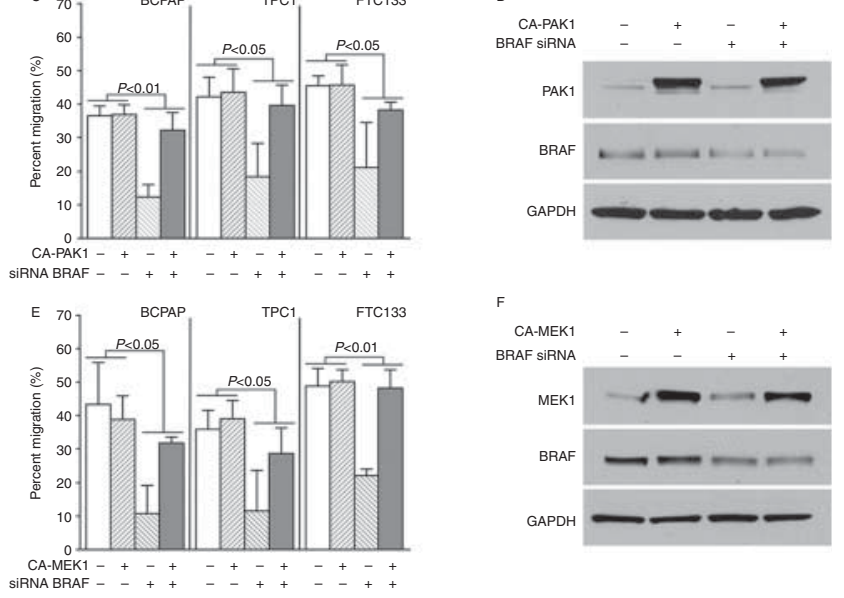

Figure 2

$B R A F$ regulates migration through PAK and MEK. (A) BRAF siRNA or scrambled sequence control was transfected into BCPAP, TPC1, and FTC133 cells. BRAF siRNA significantly reduced thyroid cancer cell migration in all three-cell lines compared with the control scrambled sequence. $P$ values are as noted in the figure. (B) MTT demonstrates no statistical difference between cells transfected with BRAF siRNA or the control scrambled sequence. (C) CA PAK1 rescued thyroid cancer cell migration in BRAF-siRNAtransfected cells with significant $P$ values as noted in the figure. (D) Western blotting confirmed a reduction in BRAF protein level by the siRNA and the transfection with the CA PAK1. Negative controls were transfection with a scrambled sequence for the siRNA and empty vector for the CA PAK1 respectively. (E) Rescue experiments using murine CA MEK1 also rescued migration of the BRAF-siRNA transfected cells in all three-thyroid cancer cell lines. The transfection efficiencies were $\sim 30-40 \%$. The $P$ values were statistically significant as noted in the figure. (F) Western blotting confirmed a reduction in BRAF protein level by the siRNA and the transfection with the CA MEK1. Negative controls were transfection with a scrambled sequence for the siRNA and empty vector for the CA MEK1 respectively.

employed in the migration assays, MTT assays were performed and showed no significant effects (Fig. 2B). To determine whether PAK1 played a role in BRAF-regulated thyroid cell migration induced by a strong serum gradient, rescue experiments were performed in all three-cell lines. CA PAK1, when expressed in BRAF siRNA-transfected thyroid cancer cells, resulted in a statistically significant rescue of migration (Fig. 2C). This effect was consistent for all three-cell lines tested when analyzed using a linear mixed statistical model. Western blotting confirmed CA PAK1 expression and BRAF knockdown (Fig. 2D). MTT assays confirmed that the transfections had minimal effects on cell viability in the conditions used for the experiments (data not shown). Similar rescue experiments using CA MEK1 demonstrated that CA MEK1 also rescued the effect of BRAF siRNA on migration, and that the rescue was consistent for all three-cell lines. A similar mixed model with an interaction effect was used as for the CA PAK1 rescue experiment (Fig. 2E). The western blotting analysis confirmed CA MEK1 expression and a partial $B R A F$ knockdown (Fig. 2F) and MTT assays confirmed that the transfections only minimally altered cell viability in the same experimental conditions (data not shown).

\section{MEK and group 1 PAK Pathways do not synergistically regulate migration}

MEK and group 1 PAKs were inhibited using U0126, a specific MEK1/2 inhibitor $(2 \mu \mathrm{M})$ or PID transfection respectively in each cell line, and migration experiments were performed. The inhibition using either U0126 or PID alone significantly reduced migration compared with the appropriate control. Inhibition of both MEK and group 1 PAKs simultaneously, however, did not result in a greater decrease in migration. These results suggest that either the two pathways are not synergistic or that they are maximally individually inhibited (Fig. 3A). The western blotting analysis confirmed a U0126-induced decrease in MEK activity, indicated by a decrease in pERK1/2 and expression of GFP-tagged PID or GFP control vector (Fig. 3B).

\section{MEK inhibition does not alter PAK activity}

Because MEK is a downstream target of BRAF that is functionally interactive with PAK (Slack-Davis et al. 2003), we performed experiments to determine whether MEK regulated PAK activity in thyroid cancer cells. MEK kinase activity was first inhibited using U0126 at increasing concentrations in BCPAP, TPC1, and FTC133 cells. U0126 did not reduce the levels of phosphorylated PAK or PAKmediated phosphorylated vimentin (Fig. 4A). Treatment with U0126 at a dose $(2.0 \mu \mathrm{M})$ in which MEK inhibition is near-complete (Fig. 4A) results in significantly reduced thyroid cancer cell motility (Fig. 4B) without reducing cell viability (Fig. 4C). This result is consistent with PAKindependent motility inhibition. We also knocked down MEK1 and MEK2 using siRNA and confirmed that loss of MEK expression does not alter PAK activity (Fig. 4D).

\section{Exogenous BRAF and PAK1 co-localize and co-IP}

To further characterize the BRAF-PAK interaction, we carried out experiments to determine if BRAF and PAK

Published by Bioscientifica Ltd 


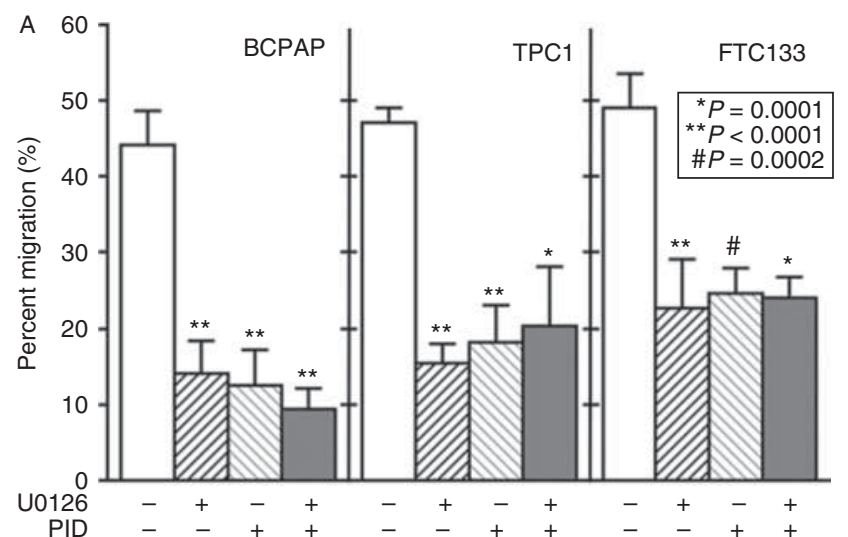

B

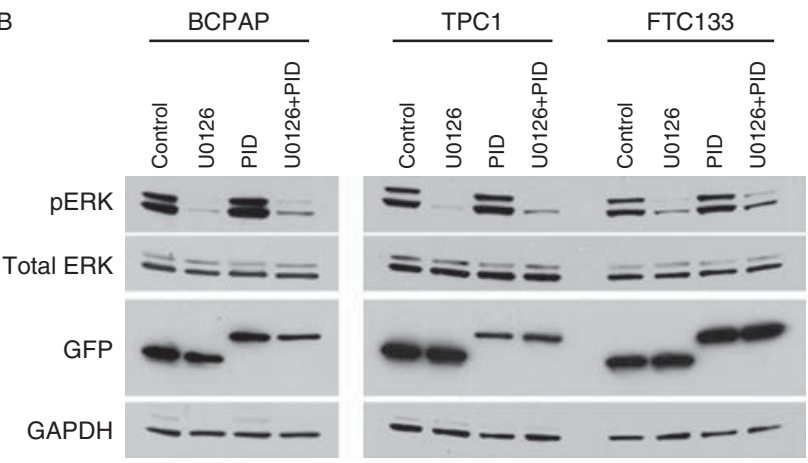

Figure 3

MEK and group 1 PAKs regulate thyroid cancer cell motility. (A) MEK1/2 and group 1 PAK activity were inhibited using U0126 $(2 \mu \mathrm{M})$ or DMSO and GFP-PID or GFP-control respectively. Migration experiments showed that inhibiting both MEK and group 1 PAKs independently and simultaneously significantly inhibited migration to a similar degree ( $P$ values as shown in figure). (B) Western blotting confirmed inhibition of MEK activity by $U 0126$ and successful transfection of the PID and control vector by immunoblot for phosphorylated ERK and GFP respectively. The transfection efficiency was $\sim 30-40 \%$ based on imaging of GFP.

co-localize and/or physically interact. Epitope tagged MYC-BRAF and FLAG-PAK1 were transfected into HEK293 cells and IP was performed using pre-conjugated FLAG, MYC, and HA (control for non-specific binding) beads. The western blotting analysis of the precipitated proteins demonstrated that BRAF and PAK1 co-IP in both directions; no non-specific pull-down was identified from the IP using pre-conjugated HA beads (Fig. 5A). To examine whether exogenous BRAF and group 1 PAKs co-localize, HEK293 cells were transfected with epitopetagged MYC-BRAF and FLAG-PAK1 cDNAs, stained with DAPI (nuclear), Alexa-488 (FLAG), and Alexa-594 (BRAF), and examined by confocal microscopy immunofluorescence (IF). In these experiments, the exogenous BRAF and PAK1 co-localize mostly in the cytosol (yellow), indicated by double arrows, while PAK alone is identified at the lamellopodia (green), indicated by single arrows (Fig. 5B).

\section{Endogenous BRAF and PAK1 co-localize and co-IP}

To determine if endogenous BRAF and PAK1 co-localize or co-IP, TPC1 and FTC133 cells with WT BRAF were stained with DAPI (nuclear), Alexa-488 (pPAK and PAK1), and Alexa-594 (BRAF) and examined by confocal microscopy. IF showed that BRAF co-localized with both PAK1 and PPAK, but this was most striking for pPAK and was particularly notable in the cytosol of mitotic cells (Fig. 6A; low power in Supplementary Fig. 1, see section on supplementary data given at the end of this article). To measure the degree of co-localization, Pearson's coefficient of correlation $(r)$ was used to analyze the confocal images to quantify a co-localization percentage value. In both cell lines, BRAF strongly co-localized with PAK1 and pPAK (Fig. 6B). Protein from TPC1 cells continuously cultured in growth medium was isolated and IP was performed. In these conditions, BRAF and PAK1 did not co-IP (Supplementary Fig. 2). As the IF suggested that BRAF and PAK co-localization occurred mostly in mitotic cells, TPC1 cells were treated for $24 \mathrm{~h}$ with $2 \mathrm{mM}$ of thymidine followed by $24 \mathrm{~h}$ with $100 \mathrm{ng} / \mathrm{ml}$ of nocodazole to increase the percentage of cells in $\mathrm{G} 2 / \mathrm{M}$ phase (Fig. 6C). IP and western blot analysis using the BRAF and PAK1 antibodies demonstrate that endogenous BRAF and PAK1 co-IP in both directions in these conditions, although it required large amounts of protein and the interaction was more strongly detected when the IP was performing using the PAK antibody (Fig. 6D).

\section{BRAFV600E expression in murine thyroids increases PAK isoform phosphorylation, activity, and expression}

To study the signaling effects of acute activation of BRAF on group 1 PAKs in vivo, we obtained paraffin blocks from the thyroid glands of mice with thyroid-specific, doxycycline-inducible BRAFV600E following 1 week of BRAFV600E overexpression that causes the development of PTC (Chakravarty et al. 2011). Compared with littermate controls, the thyroid glands from mice with induced BRAFV600E show an increase in immunoactive pPAK Thr423 (Fig. 7A). Total PAK1/2/3 protein expression was similar or increased in the mice. To confirm the immunohistochemistry staining and allow for quantitation, Western blot was performed on protein isolated from the mouse thyroid glands following BRAFV600E induction and after doxycycline treatment of age-matched control WT mice and mice expressing the Tg-rTTA

Published by Bioscientifica Ltd 


$$
\text { A }
$$

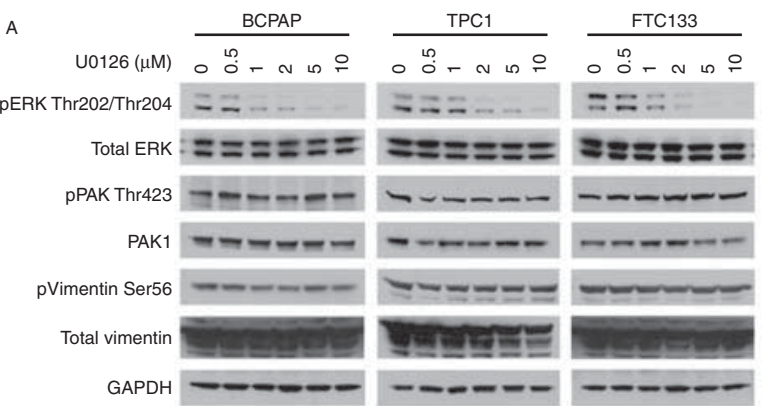

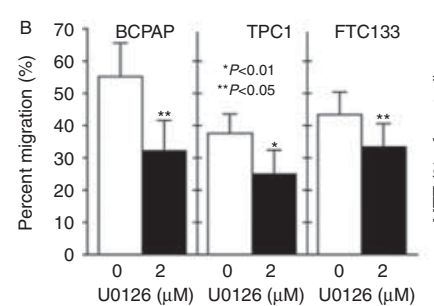
U0126 ( $\mu \mathrm{M}) \quad \mathrm{U} 0126(\mu \mathrm{M}) \quad \mathrm{U} 0126(\mu \mathrm{M})$ D

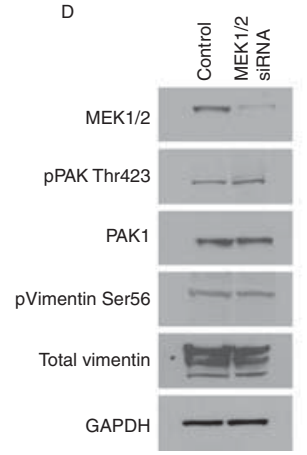

\section{Figure 4}

PAK activity is not MEK-dependent. (A) Western blotting of protein isolated from BCPAP, TPC1, and FTC133 cell lines treated with 00126 (or control DMSO) at doses sufficient to inhibit MEK kinase function did not alter PAK-mediated phosphorylation of vimentin at serine 56 . (B) $2.0 \mu \mathrm{M}$ of $\mathrm{U} 0126$ reduced thyroid cancer cell migration compared with DSMO control. Statistical significance ( $P$ values) as noted in the figure. (C) MTT assays confirmed that $2.0 \mu \mathrm{M}$ of $\mathrm{U} 0126$ in the conditions used for migration did not reduce cell viability. (D) Western blot demonstrates that a reduction of MEK $1 / 2$ levels with siRNA also did not affect pPAK or PAK1 protein levels vs cells transfected scrambled sequence control. Western blottings are representative of experiments performed on at least three independent occasions.

construct. In comparison to both controls, the mice with induced BRAFV600E had an increase in immunoactive pPAK Thr423 and pPAK Ser144, p-vimentin Ser55, and pERK consistent with activation of both PAK and MEK pathways (Fig. 7B). Interestingly, PAKs 1 and 2, and vimentin protein levels also were increased. These data suggest that acute activation of BRAF, or BRAFV600E, specifically increases both expression and signaling activation of PAK in vivo. This is consistent with our previously reported data from the invasive fronts of human PTC samples (McCarty et al. 2010). Printed in Great Britain

\section{Discussion}

In the present study, we report a functional interaction between BRAF, a critical oncogene in thyroid cancer, and PAK, a key regulator of cell motility and tumorigenesis.

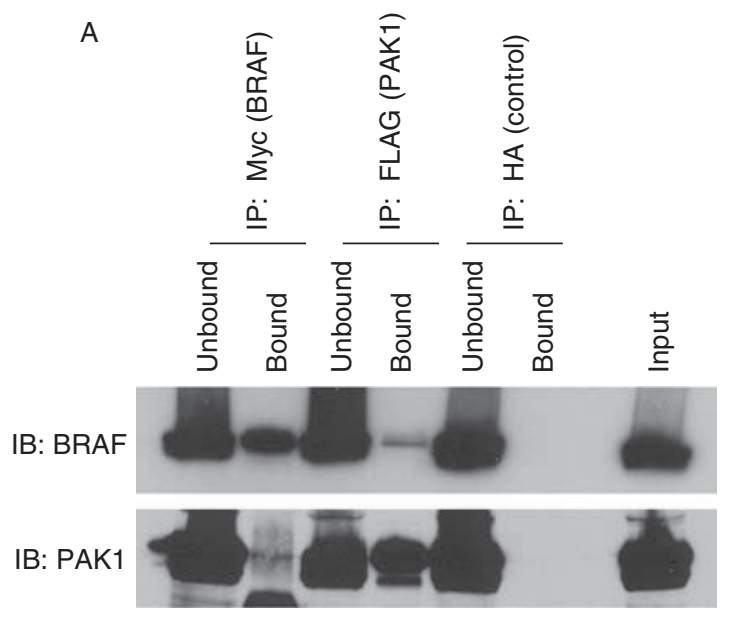

B
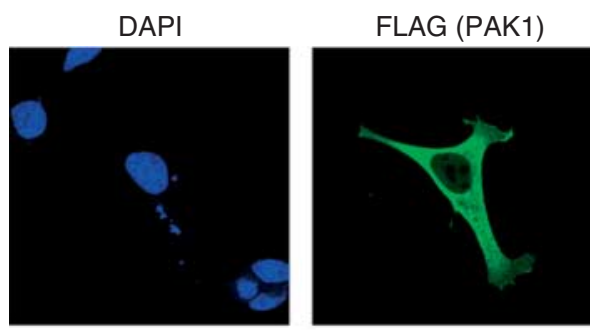

BRAF

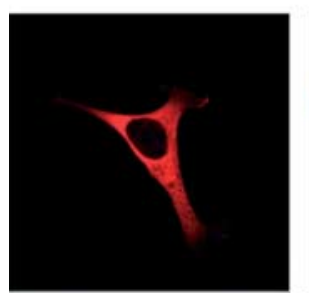

Merged

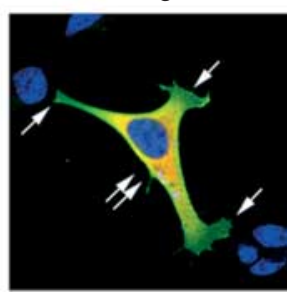

Figure 5

Exogenous BRAF and PAK1 co-immunoprecipitate (IP) and co-localize. (A) IP was performed on protein isolated from HEK293 cells that were transfected with CDNAs encoding Myc-tagged WT BRAF and FLAG-tagged WT PAK1. The IP using preconjugated anti-Myc and anti-FLAG antibodies demonstrated on immunoblot (IB) using both the epitope tag and showed that the protein was precipitated and also that a small amount of BRAF precipitated with the FLAG IP and PAK precipitated with the Myc IP. Unbound is IB of the protein in the supernatant before washing and bound is the IP protein. Input is IB of the total protein lysate serving as a size control. Non-specific binding was not identified using the protein precipitated with pre-conjugated anti-HA antibody (control). (B) Co-transfected HEK293 cells were stained with DAPI (nuclear), Alexa-488 (FLAG), and Alexa-594 (BRAF) and subjected to immunofluorescence with confocal microscopy. Imaging revealed that BRAF and PAK1 co-localized (yellow, double arrow) in the perinuclear region, but not at the lamellopodia, which contained only PAK (single arrow). Data shown are representative of experiments performed on at least three occasions.

Published by Bioscientifica Ltd 
A

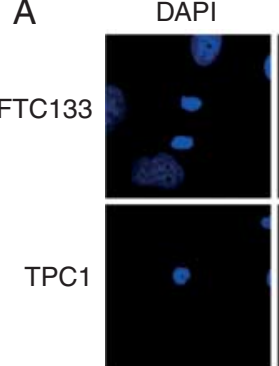

DAPI

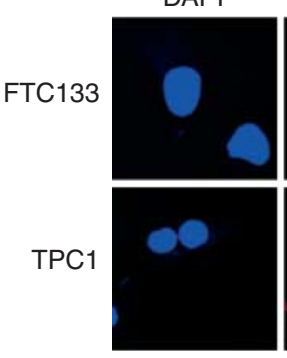

B

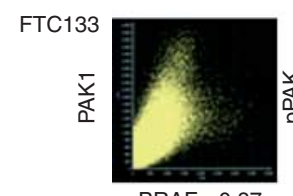

TPC

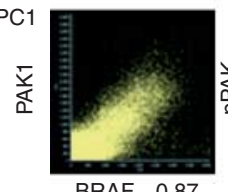

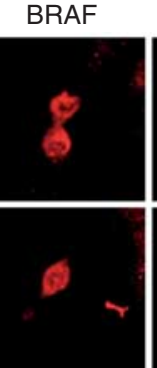

BRAF
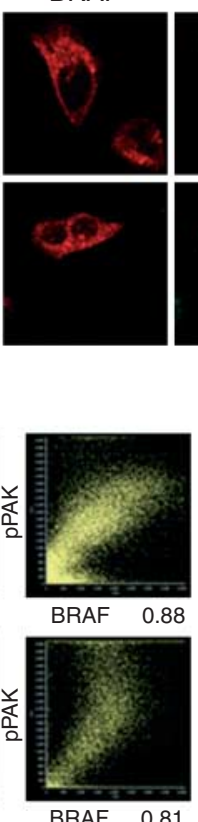

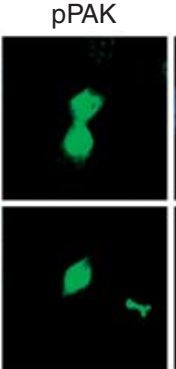

pPAK

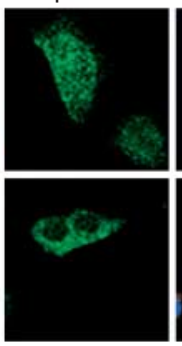

D

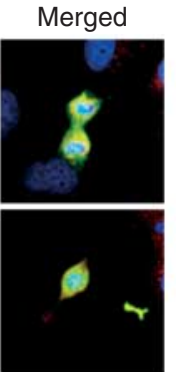

Merged

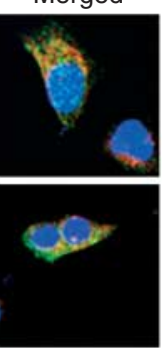

C
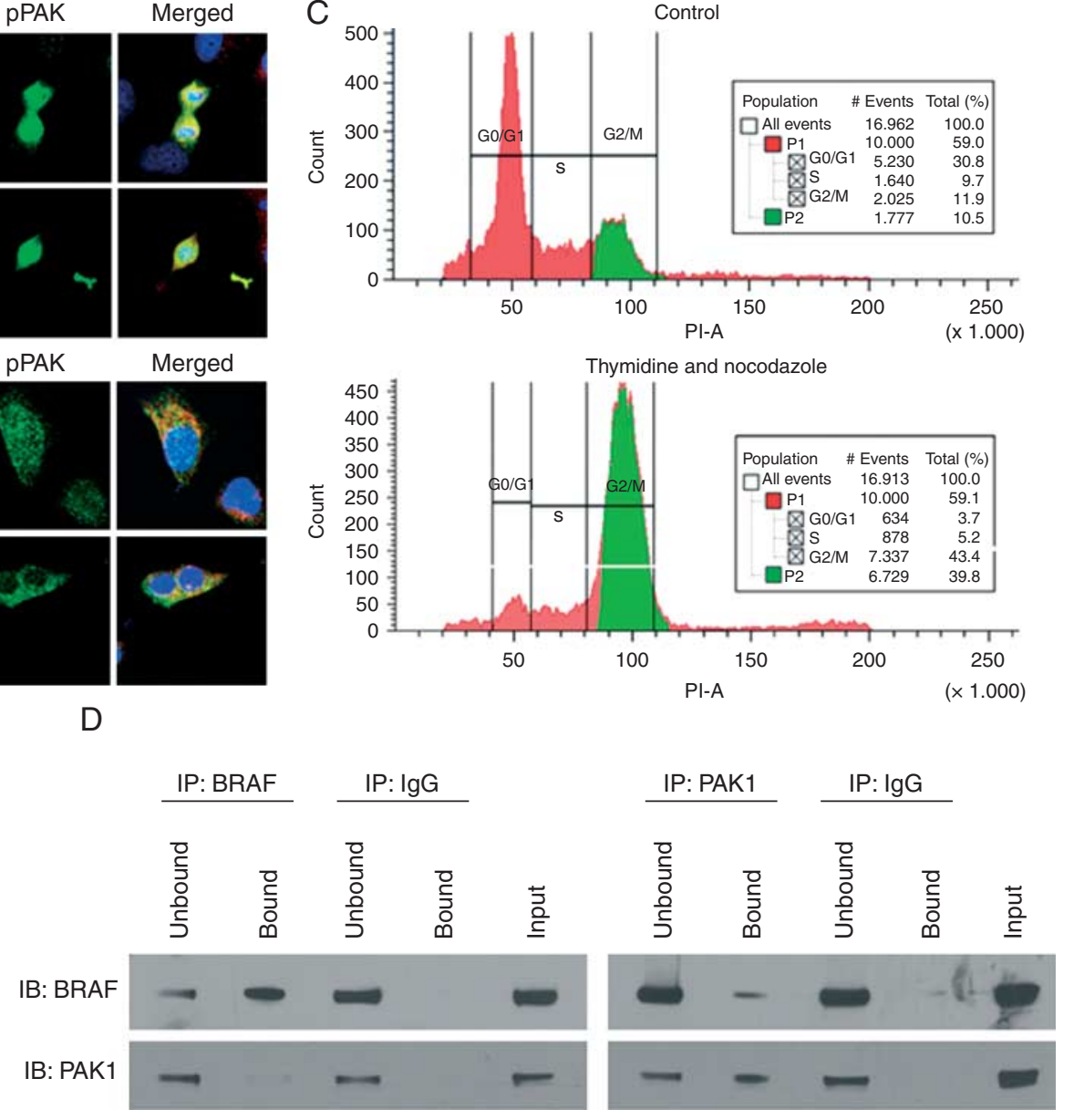

\section{Figure 6}

Endogenous BRAF and PAK1 co-immunoprecipitate (IP) and co-localize (A) TPC1 and FTC133 cells in continuous growth conditions were stained with DAPI (nuclear), Alexa-488 (PAK1 and Thr423 pPAK) and Alexa-594 (BRAF) and subjected to immunofluorescence with confocal microscopy. Imaging revealed that BRAF co-localized with PPAK and PAK1. Low-power and additional high-power images are shown in Supplementary Fig. 1. (B) In representative FTC133 cells, Person's coefficient for co-localization of BRAF and PAK 1 , and BRAF and PPAK were 0.74496 and 0.86779 with percentages of 87 and $88 \%$ respectively. In representative TPC1 cells, Person's coefficient for co-localization of BRAF and PAK1, and BRAF and PPAK, were 0.83179 and 0.75334 with percentages of 87 and $80 \%$ respectively. (C) TPC1 cells

Loss of BRAF expression in thyroid cancer cells results in reduced PAK function in vitro and BRAFV600E induction in thyroid cells in vivo leads to PAK overexpression and activation. The data further demonstrate that PAK is not regulated by MEK in thyroid cancer cells, suggesting a MEK-independent BRAF-PAK signaling connection. Finally, we demonstrate that BRAF and PAK1 physically interact in both exogenous expression and endogenous systems. Interestingly, the physical interaction between Printed in Great Britain were treated with thymidine and nocodazole, which increased the percentage of cells in G2/M phase (39.8\%) compared with the untreated control (10.5\%). (D) Protein was isolated from thymidine and nocodazoletreated TPC1 cells and IP was performed using BRAF and PAK1 antibodies, and IgG as a negative control. IB of the precipitated protein demonstrated that BRAF-precipitated protein contained PAK1, and that PAK1-precipitated protein contained BRAF with no non-specific binding with IgG. Unbound is IB of the protein in the supernatant before washing and bound is the IP protein. Input is IB of the total protein lysate to confirm size. Data shown are representative of experiments performed on at least two occasions.

endogenous WT BRAF and PAK1 appears to be most detectable in mitosis, although the precise nature of the interaction and whether it occurs as part of a larger protein complex requires further study. Recent data support the assertion of a role for $P A K$ in MAP kinase pathwaymediated tumor formation in vitro and in vivo. It was recently demonstrated that $P a k 1^{-1-}$ and $P a k 1^{+/-}$mice are relatively resistant to RAS-driven squamous cell carcinomas (Chow et al. 2012). PAK1 has been shown to

Published by Bioscientifica Ltd. 

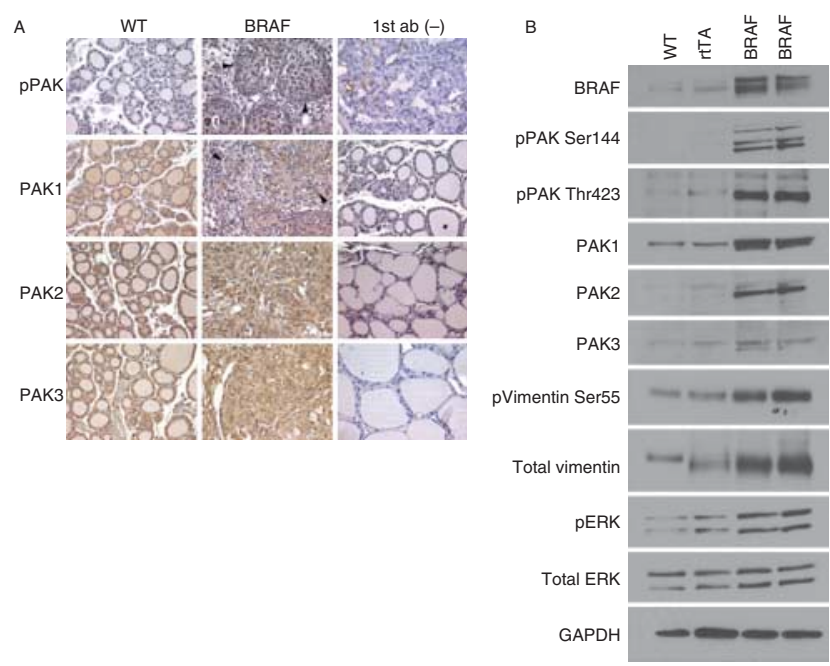

\section{Figure 7}

pPAK expression is increased by acute expression of BRAFV600E in murine thyroid glands. (A) Slide sections of thyroid glands isolated from mice following induction of thyroid-specific doxycycline-inducible BRAFV600E demonstrated increased levels of immunoactive Thr423 phosphorylated PAK compared with WT control littermates. Total levels of PAK 1/2/3 were similar between mice with the expression of BRAFV600E vs control. Negative controls (omitting the primary antibody) are shown. (B) Western blot of protein was isolated from the thyroid glands of doxycycline (dox)treated mice with thyroid-specific inducible BRAFV600E and dox-treated WT and $\mathrm{rTTA}$-expressing control mice. Induction of BRAFV600E resulted in increased levels of phosphorylated PAK, total PAKs 1 and 2, and as well as PAK and MEK-specific phosphorylation of vimentin and ERK respectively.

be particularly critical for MAPK activation in BRAF WT melanoma and in breast cancer by linking MET activation to MAPK signaling (Ong et al. 2011, Shrestha et al. 2012). This has been shown to be related to PAK-mediated phosphorylation of CRAF. In thyroid cells, even in the absence of an activating mutation, BRAF is the dominant $R A F$ isoform mediating upstream-activated oncogenic pathways (Mitsutake et al. 2006).

In all three tested thyroid cancer cell lines, two with WT BRAF (TPC1 and FTC133) and one heterozygous for the BRAFV600E mutation (BCPAP), BRAF suppression reduced PAK activity. The interaction between WT BRAF and PAK was then further explored in the co-IP and IF studies, in which interaction of the proteins was observed. The mice with acute overexpression of BRAFV600E in the thyroid display both increased expression of PAK1 and PAK2 and phosphorylation (Fig. 7B). This model is one of acute activation and overexpression and is a robust system to study acute signaling effects. Experiments in mice that stably express a single copy of BRAFV600E in the thyroid glands are ongoing. Further experiments are required to determine if the components of the BRAF/PAK protein complex, and the mechanism of BRAF-regulated PAK activity differ for BRAFV600E vs WT BRAF.

It is of interest that in human melanoma cells, the absence of BRAFV600E mutation is reported to be associated with increased PAK signaling through CRAF and to predict response to PAK inhibition (Ong et al. 2011). Moreover, in melanoma cells, the BRAFV600Especific kinase inhibitor vemurafinib did not inhibit PAK phosphorylation of MEK in cells homozygous for BRAFV600E, suggesting that that mutant BRAF may not signal through PAK as efficiently in these cells (Ong et al. 2011). However, to our knowledge, the role of WT BRAF on PAK activity or of BRAFV600E beyond pharmacological kinase inhibition has not yet been reported in melanoma. In the present study, thyroid cancer cells with WT BRAF or that are heterozygous for BRAFV600E demonstrate BRAF expression-dependent PAK activation. The difference between the two sets of results may be due to the loss of both kinase and scaffold functions in the knockdown model, the presence of WT BRAF in the thyroid systems, and/or cell autonomous differences in response to BRAF inhibitors (as has been previously described (Montero-Conde et al. 2013)). Interestingly, it has been reported recently that melanoma cells heterozygous for BRAFV600E display different responses to vemurafinib compared with cells that are homozygous for the mutant allele (Sapkota et al. 2013).

It also is important to recognize the centrality of BRAF activity in PTC tumorigenesis in the setting of activated WT BRAF (via RAS mutations and RET/PTC rearrangements for example) or through activating BRAF mutations. This is supported by the relative mutual exclusivity of these oncogenes and the importance of BRAF signaling for their oncogenic functions (Melillo et al. 2005). As the activation of PAK is known to be enhanced at the invasive fronts of tumors (Vasko \& Saji 2007), it is possible that PAK signaling plays a role in the progression of MAPK-activated PTCs in general, but the mechanisms may vary dependent on the mode of pathway activation.

While the co-IP and co-localization experiments indicate that BRAF and PAK1 physically interact, the precise nature or requirements for this interaction have not yet been elucidated. It seems likely that both proteins are part of a larger complex because BRAF signaling is facilitated through kinase suppressor of RAS 1 (KSR1) and other scaffold proteins (McKay et al. 2009). Further evidence that strengthens this possibility is that scans using STRING v9.1 (Franceschini et al. 2013) of both BRAF and PAK1 do not predict direct binding sites. Of interest are recent data demonstrating that PAK can function as a scaffold by binding signaling

Published by Bioscientifica Ltd. 
molecules to its N-terminus domain. Specifically, PAK1 was shown to directly bind to both PDK1 and AKT, and to facilitate AKT membrane recruitment and PDK1 phosphorylation (Higuchi et al. 2008). In addition, PAK scaffolding has been reported to facilitate CRAF-MEK1 signaling (Wang et al. 2013). In the present study, the inhibitor PID did not reduce the levels of pERK. This was unexpected and suggests either cell type or conditionspecific differences (low confluence vs high) from the prior studies. Thus, it is possible that PAK binds to a BRAFassociated complex leading to their functional interaction but that the precise downstream effects may depend on other components of the complex. Current studies are ongoing to determine the nature of their interaction.

Another question regarding BRAF and PAKs relationship was whether BRAF could directly phosphorylate group 1 PAKs. We evaluated this in preliminary studies using in vitro kinase assays in which PAK1 was a substrate for activated BRAF. In these experiments, PAK1 was not phosphorylated by BRAF (data not shown); however, not all PAK isoforms were evaluated and other members of the potential BRAF-PAK complex may be needed to facilitate such the event. Further evidence opposing BRAF phosphorylation of PAK comes from the Human Protein Reference Database program (Keshava Prasad et al. 2009), which did not identify a BRAF phosphorylation motif on PAK1. Taken together, it seems unlikely that PAK1 (Wang et al. 2013) is directly phosphorylated by BRAF. The lack of a cooperative effect on migration by dually inhibiting MEK and PAK, and the lack of PAK inhibition by inhibiting MEK in those same conditions, suggests that either both pathways can maximally inhibit cell motility alone or that both signaling pathways merge downstream in the thyroid cell systems that were studied. PAKs have previously been shown to regulate MEK1 by phosphorylating Ser298, thus a common downstream pathway seems possible (Slack-Davis et al. 2003). Finally, the role of other PAK isoforms on BRAF interactions has not been tested in detail to date; we focused on PAK1 due to our prior studies demonstrating that this is the primary isoform that regulates the migration of thyroid cancer cells (McCarty et al. 2010).

In summary, we have identified a functional physical interaction between BRAF and PAK1 that regulates cell motility. The presence of the pathway is supported by activation and inhibition studies, co-localization and co-IP studies, and is further supported by in vivo data. While further studies are needed to fully characterize the protein complex between BRAF and PAK, the presence of this signaling pathway is relevant for $B R A F$-mediated tumor formation, progression, and perhaps resistance to therapeutic inhibitors in thyroid cancer.

\section{Supplementary data}

This is linked to the online version of the paper at http://dx.doi.org/10.1530/ ERC-14-0424.

\section{Declaration of interest}

The authors declare that there is no conflict of interest that could be perceived as prejudicing the impartiality of the research reported.

\section{Funding}

This work was supported by the NIH Grant (P01CA124570) to M D Ringel, National Institute of General Medical Sciences of the NIH (under Award T32GM068412 to C M Knippler), and Grants P30CA016058 and S10RR025443.

\section{References}

Arozarena I, Sanchez-Laorden B, Packer L, Hidalgo-Carcedo C, Hayward R, Viros A, Sahai E \& Marais R 2011 Oncogenic BRAF induces melanoma cell invasion by downregulating the cGMP-specific phosphodiesterase PDE5A. Cancer Cell 19 45-57. (doi:10.1016/j.ccr.2010.10.029)

Beeser A \& Chernoff J 2005 Production and use of a cell permeable inhibitor of group A Paks (TAT-PID) to analyze signal transduction. Methods $\mathbf{3 7}$ 203-207. (doi:10.1016/j.ymeth.2005.05.017)

Chakravarty D, Santos E, Ryder M, Knauf JA, Liao XH, West BL, Bollag G, Kolesnick R, Thin TH, Rosen N et al. 2011 Small-molecule MAPK inhibitors restore radioiodine incorporation in mouse thyroid cancers with conditional BRAF activation. Journal of Clinical Investigation 121 4700-4711. (doi:10.1172/JCI46382)

Chow HY, Jubb AM, Koch JN, Jaffer ZM, Stepanova D, Campbell DA, Duron SG, O'Farrell M, Cai Q, Klein-Szanto AJ et al. 2012 p21-activated kinase 1 is required for efficient tumor formation and progression in a Ras-mediated skin cancer model. Cancer Research 201214. (doi:10.1158/0008-5472.CAN-12-2246)

Ding H, Yusof AM, Kothandaraman S, Saji M, Wang C, Kumar K, Milum K, Carleton M, Pan X, Ringel MD et al. 2013 Localization of CaSR antagonists in CaSR-expressing medullary thyroid cancer. Journal of Clinical Endocrinology and Metabolism 98 E1722-E1729. (doi:10.1210/ jc. 2013-1756)

Fabien N, Fusco A, Santoro M, Barbier Y, Dubois PM \& Paulin C 1994 Description of a human papillary thyroid carcinoma cell line. Morphologic study and expression of tumoral markers. Cancer $\mathbf{7 3}$ 2206-2212. (doi:10.1002/1097-0142(19940415)73:8<2206::AIDCNCR2820730828 > 3.0.CO;2-M)

Fagin JA \& Mitsiades N 2008 Molecular pathology of thyroid cancer: diagnostic and clinical implications. Best Practice \& Research. Clinical Endocrinology \& Metabolism 22 955-969. (doi:10.1016/j.beem. 2008.09.017)

Flaiz C, Chernoff J, Ammoun S, Peterson JR \& Hanemann CO 2009 PAK kinase regulates Rac GTPase and is a potential target in human schwannomas. Experimental Neurology 218 137-144. (doi:10.1016/ j.expneurol.2009.04.019)

Franceschini A, Szklarczyk D, Frankild S, Kuhn M, Simonovic M, Roth A, Lin J, Minguez P, Bork P, von Mering C et al. 2013 STRING v9.1: protein-protein interaction networks, with increased coverage and 
integration. Nucleic Acids Research 41 D808-D815. (doi:10.1093/nar/ gks1094)

French A, Mills S, Swarup R, Bennett M \& Pridmore T 2008 Colocalization of fluorescent markers in confocal microscope images of plant cells. Nature Protocols 3 619-628. (doi:10.1038/nprot.2008.31)

Goretzki PE, Frilling A, Simon D \& Roeher HD 1990 Growth regulation of normal thyroids and thyroid tumors in man. Recent Results in Cancer Research 118 48-63. (doi:10.1007/978-3-642-83816-3_6)

Higuchi M, Onishi K, Kikuchi C \& Gotoh Y 2008 Scaffolding function of PAK in the PDK1-Akt pathway. Nature Cell Biology 10 1356-1364. (doi:10.1038/ncb1795)

Huang W \& Erikson RL 1994 Constitutive activation of Mek1 by mutation of serine phosphorylation sites. PNAS 91 8960-8963. (doi:10.1073/ pnas.91.19.8960)

Keshava Prasad TS, Kandasamy K, Keerthikumar S, Kumar S, Mathivanan S, Telikicherla D, Raju R, Shafreen B, Venugopal A, Balakrishnan L et al. 2009 Human Protein Reference Database - 2009 update. Nucleic Acids Research 37 767-772. (doi:10.1093/nar/gkn892)

Kloos RT, Ringel MD, Knopp MV, Hall NC, King M, Stevens R, Liang J, Wakely PE Jr, Vasko VV, Saji M et al. 2009 Phase II trial of sorafenib in metastatic thyroid cancer. Journal of Clinical Oncology 27 1675-1684. (doi:10.1200/JCO.2008.18.2717)

Knauf JA, Ma X, Smith EP, Zhang L, Mitsutake N, Liao XH, Refetoff S, Nikiforov YE, Fagin JA, Mitsutake N et al. 2005 Targeted expression of BRAFV600E in thyroid cells of transgenic mice results in papillary thyroid cancers that undergo dedifferentiation. Cancer Research 65 4238-4245. (doi:10.1158/0008-5472.CAN-05-0047)

Knauf JA, Sartor MA, Medvedovic M, Lundsmith E, Ryder M, Salzano M, Nikiforov YE, Giordano TJ, Ghossein RA \& Fagin JA 2011 Progression of BRAF-induced thyroid cancer is associated with epithelial-mesenchymal transition requiring concomitant MAP kinase and TGF $\beta$ signaling. Oncogene 30 3153-3163. (doi:10.1038/onc.2011.44)

Kurebayashi J, Tanaka K, Otsuki T, Moriya T, Kunisue H, Uno M \& Sonoo H 2000 All-trans-retinoic acid modulates expression levels of thyroglobulin and cytokines in a new human poorly differentiated papillary thyroid carcinoma cell line, KTC-1. Journal of Clinical Endocrinology and Metabolism 85 2889-2896. (doi:10.1210/jcem.85.8.6732)

Landa I, Ganly I, Chan TA, Mitsutake N, Matsuse M, Ibrahimpasic T, Ghossein RA \& Fagin JA 2013 Frequent somatic TERT promoter mutations in thyroid cancer: higher prevalence in advanced forms of the disease. Journal of Clinical Endocrinology and Metabolism 98 E1562-E1566. (doi:10.1210/jc.2013-2383)

Lei M, Robinson MA \& Harrison SC 2005 The active conformation of the PAK1 kinase domain. Structure 13 769-778. (doi:10.1016/j.str.2005. 03.007)

Liu X, Qu S, Liu R, Sheng C, Shi X, Zhu G, Murugan AK, Guan H, Yu H, Wang Y et al. 2014 TERT promoter mutations and their association with BRAF V600E mutation and aggressive clinicopathological characteristics of thyroid cancer. Journal of Clinical Endocrinology and Metabolism 99 E1130-E1136. (doi:10.1210/jc.2013-4048)

Ma QL, Yang F, Frautschy SA \& Cole GM 2012 PAK in Alzheimer disease, Huntington disease and X-linked mental retardation. Cellular Logistics 2 117-125. (doi:10.4161/cl.21602)

Makrodouli E, Oikonomou E, Koc M, Andera L, Sasazuki T, Shirasawa S \& Pintzas A 2011 BRAF and RAS oncogenes regulate Rho GTPase pathways to mediate migration and invasion properties in human colon cancer cells: a comparative study. Molecular Cancer 10 118. (doi:10.1186/14764598-10-118)

McCarty SK, Saji M, Zhang X, Jarjoura D, Fusco A, Vasko VV \& Ringel MD 2010 Group I p21-activated kinases regulate thyroid cancer cell migration and are overexpressed and activated in thyroid cancer invasion. Endocrine-Related Cancer 17 989-999. (doi:10.1677/ERC10-0168)

McKay MM, Ritt DA \& Morrison DK 2009 Signaling dynamics of the KSR1 scaffold complex. PNAS 106 11022-11027. (doi:10.1073/pnas. 0901590106)
Melillo RM, Castellone MD, Guarino V, De Falco V, Cirafici AM, Salvatore G, Caiazzo F, Basolo F, Giannini R, Kruhoffer M et al. 2005 The RET/PTC-RAS-BRAF linear signaling cascade mediates the motile and mitogenic phenotype of thyroid cancer cells. Journal of Clinical Investigation 115 1068-1081. (doi:10.1172/ JCI200522758)

Mitsutake N, Miyagishi M, Mitsutake S, Akeno N, Mesa C Jr, Knauf JA, Zhang L, Taira K \& Fagin JA 2006 BRAF mediates RET/PTC-induced mitogen-activated protein kinase activation in thyroid cells: functional support for requirement of the RET/PTC-RAS-BRAF pathway in papillary thyroid carcinogenesis. Endocrinology 147 1014-1019. (doi:10.1210/en.2005-0280)

Montero-Conde C, Ruiz-Llorente S, Dominguez JM, Knauf JA, Viale A, Sherman EJ, Ryder M, Ghossein RA, Rosen N \& Fagin JA 2013 Relief of feedback inhibition of HER3 transcription by RAF and MEK inhibitors attenuates their antitumor effects in BRAF-mutant thyroid carcinomas. Cancer Discovery 3 520-533. (doi:10.1158/2159-8290. CD-12-0531)

Nucera C, Lawler J \& Parangi S 2011 BRAFV600E and microenvironment in thyroid cancer: a functional link to drive cancer progression. Cancer Research 71 2417-2422. (doi:10.1158/0008-5472.CAN10-3844)

Ong CC, Jubb AM, Haverty PM, Zhou W, Tran V, Truong T, Turley H, O'Brien T, Vucic D, Harris AL et al. 2011 Targeting p21-activated kinase 1 (PAK1) to induce apoptosis of tumor cells. PNAS $\mathbf{1 0 8} 7177-7182$. (doi:10.1073/pnas.1103350108)

Pellegriti G, Frasca F, Regalbuto C, Squatrito S \& Vigneri R 2013 Worldwide increasing incidence of thyroid cancer: update on epidemiology and risk factors. Journal of Cancer Epidemiology 2013 965212. (doi:10.1155/ 2013/965212)

Porchia LM, Guerra M, Wang YC, Zhang Y, Espinosa AV, Shinohara M, Kulp SK, Kirschner LS, Saji M, Chen CS et al. 2007 2-Amino-N-\{4-[5(2-phenanthrenyl)-3-(trifluoromethyl)-1H-pyrazol-1-yl]-phenyl\}acetamide (OSU-03012), a celecoxib derivative, directly targets p21-activated kinase. Molecular Pharmacology 72 1124-1131. (doi:10.1124/mol.107. 037556)

Radu M, Semenova G, Kosoff R \& Chernoff J 2014 PAK signalling during the development and progression of cancer. Nature Reviews. Cancer 14 13-25. (doi:10.1038/nrc3645)

Rider LF, Oladimeji P \& Diakonova M 2013 PAK1 regulates breast cancer cell invasion through secretion of matrix metalloproteinases in response to prolactin and three-dimensional collagen IV. Molecular Endocrinology 27 1048-1064. (doi:10.1210/me.2012-1322)

Riesco-Eizaguirre G, Rodriguez I, De la Vieja A, Costamagna E, Carrasco N, Nistal M \& Santisteban P 2009 The BRAFV600E oncogene induces transforming growth factor \{beta\} secretion leading to sodium iodide symporter repression and increased malignancy in thyroid cancer. Cancer Research 69 8317-8325. (doi:10.1158/0008-5472.CAN-09-1248)

Ringel MD 2009 Molecular markers of aggressiveness of thyroid cancer. Current Opinion in Endocrinology, Diabetes, and Obesity 16 361-366. (doi:10.1097/MED.0b013e32832ff2cb)

Ringel MD, Hayre N, Saito J, Saunier B, Schuppert F, Burch H, Bernet V, Burman KD, Kohn LD \& Saji M 2001 Overexpression and overactivation of Akt in thyroid carcinoma. Cancer Research 61 6105-6111.

Sapkota B, Hill CE \& Pollack BP 2013 Vemurafenib enhances MHC induction in BRAF homozygous melanoma cells. Oncoimmunology 2 e22890. (doi:10.4161/onci.22890)

Schweppe RE, Klopper JP, Korch C, Pugazhenthi U, Benezra M, Knauf JA, Fagin JA, Marlow L, Copland JA, Smallridge RC et al. 2008 Deoxyribonucleic acid profiling analysis of 40 human thyroid cancer cell lines reveals cross-contamination resulting in cell line redundancy and misidentification. Journal of Clinical Endocrinology and Metabolism $\mathbf{8 3}$ 4331-4341. (doi:10.1210/jc.2008-1102)

Shrestha Y, Schafer EJ, Boehm JS, Thomas SR, He F, Du J, Wang S, Barretina J, Weir BA, Zhao JJ et al. 2012 PAK1 is a breast cancer oncogene that 
coordinately activates MAPK and MET signaling. Oncogene $\mathbf{3 1}$ 3397-3408. (doi:10.1038/onc.2011.515)

Slack-Davis J, Eblen S, Zecevic M, Boerner S, Tarcsafalvi A, Diaz H, Marshall M, Weber M, Parsons J \& Catling A 2003 PAK1 phosphorylation of MEK1 regulates fibronectin-stimulated MAPK activation. Journal of Cell Biology 162 281-291. (doi:10.1083/jcb.200212141)

Vasko VV \& Saji M 2007 Molecular mechanisms involved in differentiated thyroid cancer invasion and metastasis. Current Opinion in Oncology 19 11-17. (doi:10.1097/CCO.0b013e328011ab86)

Vasko V, Espinosa AV, Scouten W, He H, Auer H, Liyanarachchi S, Larin A, Savchenko V, Francis GL, de la Chapelle A et al. 2007 Gene expression and functional evidence of epithelial-to-mesenchymal transition in papillary thyroid carcinoma invasion. PNAS 104 2803-2808. (doi:10.1073/pnas.0610733104)

Wang Z, Fu M, Wang L, Liu J, Li Y, Brakebusch C \& Mei Q 2013 p21-Activated kinase 1 (PAK1) can promote ERK activation in a kinase-independent manner. Journal of Biological Chemistry $\mathbf{2 8 8}$ 20093-20099. (doi:10.1074/jbc.M112.426023)
Whale A, Hashim FN, Fram S, Jones GE \& Wells CM 2011 Signalling to cancer cell invasion through PAK family kinases. Frontiers in Bioscience 16 849-864. (doi:10.2741/3724)

Wong RM, Bresee C \& Braunstein GD 2013 Comparison with published systems of a new staging system for papillary and follicular thyroid carcinoma. Thyroid 23 566-574. (doi:10.1089/thy.2012.0181)

Xing M 2007 BRAF mutation in papillary thyroid cancer: pathogenic role, molecular bases, and clinical implications. Endocrine Reviews $\mathbf{2 8}$ 742-762. (doi:10.1210/er.2007-0007)

Xing M, Alzahrani AS, Carson KA, Viola D, Elisei R, Bendlova B, Yip L, Mian C, Vianello F, Tuttle RM et al. 2013 Association between BRAF V600E mutation and mortality in patients with papillary thyroid cancer. Journal of the American Medical Association 309 1493-1501. (doi:10.1001/jama.2013.3190)

Zinchuk V, Zinchuk O \& Okada T 2007 Quantitative colocalization analysis of multicolor confocal immunofluorescence microscopy images: pushing pixels to explore biological phenomena. Acta Histochemica et Cytochemica 40 101-111. (doi:10.1267/ahc.07002)

Received in final form 10 September 2014

Accepted 15 September 2014

Made available online as an Accepted Preprint

16 September 2014
(C) 2014 Society for Endocrinology Printed in Great Britain
Published by Bioscientifica Ltd. 